\title{
Asymmetric silicate dust distribution toward the silicate carbon star BM Geminorum ${ }^{\star}$
}

\author{
K. Ohnaka ${ }^{1}$, H. Izumiura ${ }^{2}$, Ch. Leinert ${ }^{3}$, T. Driebe $^{1}$, G. Weigelt ${ }^{1}$, and M. Wittkowski ${ }^{4}$ \\ 1 Max-Planck-Institut für Radioastronomie, Auf dem Hügel 69, 53121 Bonn, Germany \\ e-mail: kohnaka@mpifr-bonn.mpg.de \\ 2 Okayama Astrophysical Observatory, National Astronomical Observatory, Kamogata, Asakuchi, Okayama 719-0232, Japan \\ 3 Max-Planck-Institut für Astronomie, Königstuhl 17, 69117 Heidelberg, Germany \\ ${ }^{4}$ European Southern Observatory, Karl-Schwarzschild-Str. 2, 85748 Garching, Germany
}

Received 20 May 2008 / Accepted 9 July 2008

\begin{abstract}
Context. Despite their carbon-rich photospheres, silicate carbon stars show $10 \mu \mathrm{m}$ silicate emission. They are considered to have circumbinary or circum-companion disks, which serve as a reservoir of oxygen-rich material shed by mass loss in the past. Aims. We present $\mathrm{N}$-band spectro-interferometric observations of the silicate carbon star BM Gem using MIDI at the Very Large Telescope Interferometer (VLTI). Our aim is to probe the spatial distribution of oxygen-rich dust at high spatial resolution. Methods. Using the UT2-UT3 and UT3-UT4 configurations, BM Gem was observed with VLTI/MIDI at 44-62 m baselines. Results. The $N$-band visibilities observed for BM Gem decrease steeply between 8 and $\sim 10 \mu \mathrm{m}$ and increase gradually longward of $\sim 10 \mu \mathrm{m}$, reflecting the optically thin silicate emission feature emanating from sub-micron-sized amorphous silicate grains. The differential phases obtained at baselines of $\sim 44-46 \mathrm{~m}$ show significant non-zero values $\left(\sim-70^{\circ}\right)$ in the central part of the silicate emission feature between $\sim 9$ and $11 \mu \mathrm{m}$, revealing a photocenter shift and the asymmetric nature of the silicate emitting region. The observed $\mathrm{N}$-band visibilities and differential phases can be described adequately by a simple geometrical model in which the unresolved star is surrounded by a ring with azimuthal brightness modulation. The best-fit model is characterized by a broad ring ( $\sim 70$ mas across at $10 \mu \mathrm{m}$ ) with a bright region offset from the unresolved star by $\sim 20$ mas at a position angle of $\sim 280^{\circ}$. This model can be interpreted as a system with a circum-companion disk and is consistent with the spectroscopic signatures of an accretion disk around an unseen companion, which were discovered in the violet spectrum of BM Gem.
\end{abstract}

Key words. infrared: stars - techniques: interferometric - stars: circumstellar matter - stars: carbon - stars: AGB and post-AGB stars: individual: BM Gem

\section{Introduction}

Despite their carbon-rich photospheres, "silicate carbon stars" are characterized by oxygen-rich circumstellar material, which is responsible for prominent silicate emission (Little-Marenin 1986; Willems \& de Jong 1986). The detection of masers from O-bearing molecules, such as $\mathrm{OH}$ and $\mathrm{H}_{2} \mathrm{O}$, toward some of the silicate carbon stars confirms the presence of oxygen-rich circumstellar gas (e.g. Nakada et al. 1987; Engels 1994; Szymczak et al. 2001, and references therein). Optical spectroscopic studies (e.g. Lloyd-Evans 1990; Chan 1993; Ohnaka \& Tsuji 1999) reveal that silicate carbon stars have ${ }^{12} \mathrm{C} /{ }^{13} \mathrm{C}$ ratios as low as $\sim 4-5$ (classified as "J-type"), which is difficult to explain with the present stellar evolution theory. Silicate carbon stars are considered to have an unseen, low-luminosity companion. The oxygenrich material that was shed by mass loss when the primary star was an $\mathrm{M}$ giant can be stored in a circumbinary disk (Morris 1987; Lloyd-Evans 1990) or in a circumstellar disk around the companion ("circum-companion disk", Yamamura et al. 2000), even after the primary star becomes a carbon star. In the latter case, Yamamura et al. (2000) suggest that the primary star drives an outflow from the circum-companion disk and the silicate emission emanates from this outflow.

* Based on observations made with the Very Large Telescope Interferometer of the European Southern Observatory. Program ID: 078.D-0292.
Due to progress in high-angular resolution observation techniques, there is growing observational evidence for the presence of circumbinary or circum-companion disks around silicate carbon stars. Our $N$-band spectro-interferometric observations of IRAS 08002-3803 with the MID-infrared Interferometric instrument (MIDI) at ESO's Very Large Telescope Interferometer (VLTI) spatially resolved the dusty environment of a silicate carbon star for the first time (Ohnaka et al. 2006), and our radiative transfer modeling suggests the presence of an optically thick circumbinary disk in which amorphous silicate and a second grain species (amorphous carbon, large silicate grains, or metallic iron) coexist. The presence of a circumbinary disk with multiple grain populations is also suggested for another silicate carbon star IRAS $18006-3213$ by Deroo et al. (2007). The spatial distributions of the $22 \mathrm{GHz} \mathrm{H}_{2} \mathrm{O}$ maser emission toward two silicate carbon stars, V778 Cyg and EU And, were resolved by Szczerba et al. (2006), Engels (priv. comm.), and Ohnaka \& Boboltz (2008) using MERLIN and the Very Long Baseline Array (VLBA). The observed spatio-kinematic structures are consistent with the presence of circum-companion disks. However, the formation mechanisms of these disks are poorly understood, and the possible link between disk formation and the anomalously low ${ }^{12} \mathrm{C} /{ }^{13} \mathrm{C}$ ratios is by no means clear.

Direct detection of a companion toward a silicate carbon star is difficult because of the enormous luminosity contrast between the primary carbon star $\left(10^{3}-10^{4} L_{\odot}\right)$ and its companion 
Table 1. MIDI observations of BM Gem: night, time of observation (Coordinated Universal Time = UTC), telescope configuration (Tel.), projected baseline length $B_{\mathrm{p}}$, position angle of the projected baseline on the sky (PA), and seeing in the visible at the times of the observations of BM Gem and the calibrators listed in Table 2.

\begin{tabular}{|c|c|c|c|c|c|c|}
\hline \# & Night & $\begin{array}{c}t_{\mathrm{obs}} \\
\text { (UTC) }\end{array}$ & Tel. & $\begin{array}{r}B_{\mathrm{p}} \\
(\mathrm{m})\end{array}$ & $\begin{array}{l}\text { PA } \\
\left(^{\circ}\right)\end{array}$ & Seeing \\
\hline 1 & 2006 Dec. 29 & $07: 29: 47$ & UT3-4 & 45.7 & 93.1 & $1^{\prime \prime} 0-1^{\prime \prime} 5$ \\
\hline 2 & 2006 Dec. 31 & $06: 15: 26$ & UT3-4 & 55.2 & 99.1 & $0.7-1^{\prime \prime} 0$ \\
\hline 3 & 2007 Jan. 04 & $06: 31: 59$ & UT2-3 & 43.8 & 49.8 & 0.8 \\
\hline 4 & 2007 Jan. 05 & $04: 17: 10$ & UT3-4 & 62.1 & 108.0 & $0.7-1^{\prime \prime} 0$ \\
\hline
\end{tabular}

Table 2. MIDI observations of calibrators: $12 \mu \mathrm{m}$ fluxes $\left(F_{12}\right)$, uniformdisk diameters $\left(d_{\mathrm{UD}}\right)$, night, and the time stamp $\left(t_{\mathrm{obs}}\right)$. The uniform-disk diameters were taken from the CalVin list available at ESO (http:// www. eso.org/observing/etc/). The data set used for spectrophotometric calibration is marked with $\dagger$.

\begin{tabular}{rrrcc}
\hline \hline Calibrator & $\begin{array}{r}F_{12} \\
(\mathrm{Jy})\end{array}$ & $\begin{array}{r}d_{\mathrm{UD}} \\
(\mathrm{mas})\end{array}$ & Night & $t_{\text {obs }}(\mathrm{UTC})$ \\
\hline HD48433 & 8.5 & $2.10 \pm 0.13$ & 2006 Dec. 29 & $07: 52: 09$ \\
& & & 2006 Dec. 31 & $06: 42: 34^{\dagger}$ \\
& & & 2007 Jan. 04 & $06: 08: 26$ \\
HD47205 & 9.7 & $2.30 \pm 0.12$ & 2007 Jan. 05 & $03: 41: 13$ \\
HD50778 & 24.6 & $3.95 \pm 0.22$ & 2006 Dec. 31 & $01: 35: 21$ \\
& & & 2006 Dec. 31 & $05: 35: 01$ \\
HD98430 & 19.1 & $3.09 \pm 0.16$ & 2007 Jan. 04 & $07: 03: 45$ \\
\hline
\end{tabular}

(§1 $\left.L_{\odot}\right)$, as well as small angular separations (e.g., Engels \& Leinert 1994). However, Izumiura (2003) and Izumiura et al. (2008) overcame the first problem by taking advantage of the fact that the violet flux of a carbon star (primary star) is suppressed heavily due to molecular and atomic absorption. Using the High Dispersion Spectrograph (HDS) on the Subaru Telescope, they discovered continuum emission below $4000 \AA$ and Balmer lines showing P Cygni profiles with an outflow velocity of $\gtrsim 400 \mathrm{~km} \mathrm{~s}^{-1}$ toward the silicate carbon star BM Gem. These spectroscopic signatures are consistent with the presence of an accretion disk around an unseen companion, although the companion could not be spatially resolved, and its nature - whether it is a main-sequence star or a white dwarf - remains unknown. Comparison between the observationally derived accretion luminosity $\left(\sim 0.2 L_{\odot}\right)$ and the theoretical prediction from the Bondi-Hoyle-type accretion resulted in estimated binary separations of 3-60 AU for a main-sequence star companion and 100-500 AU for a white dwarf companion. These values translate into angular separations of 2.5-50 mas and 83-420 mas for a distance of $1.2 \mathrm{kpc}$ adopted for BM Gem by Izumiura et al. (2008).

Given these estimated separations, it is challenging to resolve the circum-companion disk toward BM Gem with a singledish telescope. On the other hand, infrared interferometry provides us with a unique opportunity to achieve sufficiently high spatial resolution. In this paper, we present the results of midinfrared spectro-interferometric observations of BM Gem with VLTI/MIDI to probe the presence of the circum-companion disk with dust thermal emission.

\section{Observations}

Our MIDI observations of BM Gem and the calibrators are summarized in Tables 1 and 2, respectively. BM Gem was observed in the HIGH_SENS mode using a prism $(\lambda / \Delta \lambda \simeq 30$ at $10 \mu \mathrm{m})$, and four data sets were taken using the UT2-UT3 and UT3-UT4 configurations with projected baseline lengths of 44-62 m. A detailed description of the instrument and the observing procedure is given in Przygodda et al. (2003), Leinert et al. (2004), and Chesneau et al. (2005). We used the MIA + EWS package ver. $1.5^{1}$ to reduce the MIDI data (Leinert et al. 2004; Jaffe 2004). While both MIA and EWS can derive the visibility amplitude, EWS can also extract the so-called "differential phase", which contains information about the wavelength dependence of the object's phase. However, it should be kept in mind that two pieces of information about the object's phase are lost in the derivation of differential phase as described by Jaffe (2004): the absolute phase offset and the phase gradient with respect to wavenumber.

We assessed the data quality and derived the calibrated visibilities as described by Ohnaka et al. (2008). Since it is difficult to estimate the errors in the calibrated visibilities properly from only 1-3 calibrator measurements, we assumed a total relative error of $15 \%$ as adopted in Ohnaka et al. (2008). The visibilities obtained with MIA and EWS agree well, and we only show the results derived with EWS in the discussion below. We also extracted the absolutely calibrated $N$-band spectrum of BM Gem from our MIDI data as described in Ohnaka et al. (2007). A calibrator observed at airmass similar to our target was used for the spectrophotometric calibration and is marked with $\dagger$ in Table 2. The error of the absolutely calibrated MIDI spectrum is difficult to estimate properly with only one calibrator; we therefore assumed errors of 10-20\% as in Ohnaka et al. (2007).

\section{Results}

Figure 1a shows the MIDI spectrum of BM Gem, and its IRAS Low Resolution Spectrum (LRS) ${ }^{2}$ and photometric data from the Midcourse Space Experiment (MSX, Egan et al. 2003) and IRAS Point Source Catalog (PSC). The MIDI spectrum and IRAS/LRS show that the flux level has changed little over the past 23 years, and flux variation longward of $13 \mu \mathrm{m}$ is absent, as can be seen from the IRAS/LRS and MSX data taken in 1996-1997. This stability in the mid-infrared flux is the same as found for V778 Cyg (Yamamura et al. 2000), IRAS 08002-3803 (Ohnaka et al. 2006), IRAS 18006-3213 (Deroo et al. 2007), and EU And (Ohnaka \& Boboltz 2008).

In Fig. 1b, we also plot the spectrum of V778 Cyg obtained with the Short Wavelength Spectrometer (SWS) onboard the Infrared Space Observatory (ISO) presented in Yamamura et al. (2000) as well as those of IRAS 08002-3803 and IRAS 18006-3213 obtained with MIDI by Ohnaka et al. (2006) and Deroo et al. (2007), respectively. BM Gem and V778 Cyg show a well-defined peak at $\sim 9.8 \mu \mathrm{m}$, while IRAS 080023803 and IRAS 18006-3213 show broader features. Yamamura et al. (2000) demonstrate that the silicate feature of V778 Cyg is characterized by optically thin emission from sub-micronsized amorphous silicate grains. The close resemblance between BM Gem and V778 Cyg indicates the predominance of these small, amorphous silicate grains around BM Gem, and the silicate dust toward this object is optically thin. This is also consistent with the spectral energy distribution (SED) of BM Gem plotted in Fig. 1c, which shows only a modest infrared excess longward of $\sim 8 \mu \mathrm{m}$. As Yamamura et al. (2000) argue, such

\footnotetext{
1 Available at http://www . strw. leidenuniv.nl/ nevec/MIDI 2 Downloaded from http://staff.gemini.edu/ kvolk/ getlrs_plot.html with the correction of the absolute calibration by Cohen et al. (1992).
} 

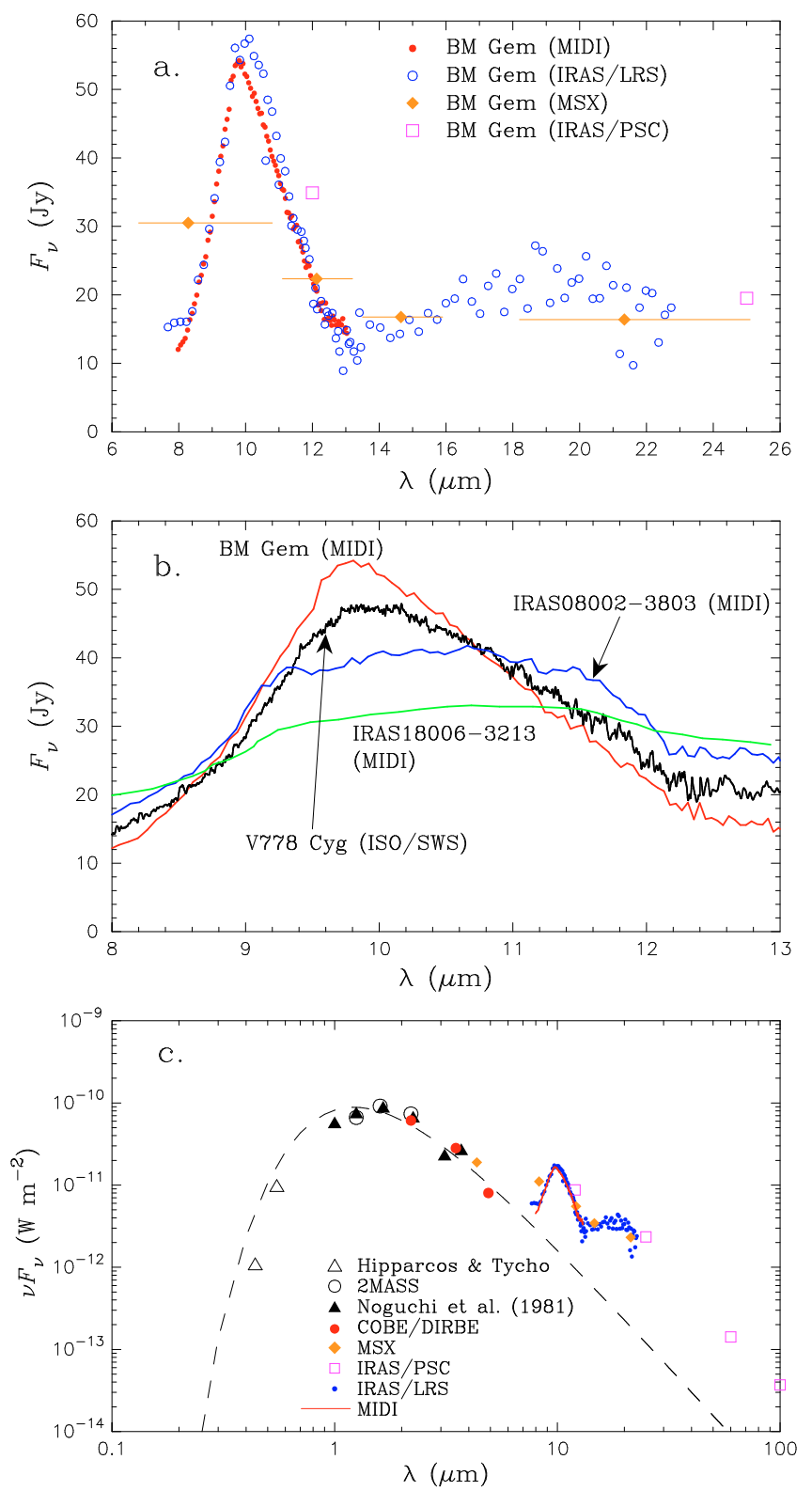

Fig. 1. a) $N$-band spectrum of BM Gem extracted from the MIDI data, plotted with IRAS/LRS as well as the photometric data from the MSX and IRAS PSC catalogs. The width of each MSX band is shown by the horizontal bar. b) Comparison of the $N$-band spectra of four silicate carbon stars: BM Gem, V778 Cyg, IRAS 08002-3803, and IRAS 18006-3213. c) SED of BM Gem constructed from the (spectro)photometric data available in the literature (Hipparcos \& Tycho Catalogs; ESA 1997; 2MASS, Cutri et al. 2003; Noguchi et al. 1981; COBE/DIRBE, Smith et al. 2004; MSX; IRAS/PSC; IRAS/LRS; MIDI). The COBE/DIRBE data longward of $12 \mu \mathrm{m}$ are not used, because of the large errors. The dashed line represents the stellar flux contribution, which is approximated with a blackbody of $3000 \mathrm{~K}$.

small grains in an optically thin environment are blown away by the radiation pressure of the primary (carbon-rich) star, suggesting that a circumbinary (or circum-primary) disk cannot exist for a long period of time. This argument is in line with our interpretation of the $N$-band visibilities and differential phases presented in Sect. 5.

On the other hand, the broad $N$-band spectra of IRAS 08002-3803 and IRAS 18006-3213 indicate the presence of grain species other than small silicate. In particular, featureless dust emission from large grains, amorphous carbon, metallic iron, or iron sulfide is suggested for both objects (Ohnaka et al. 2006; Deroo et al. 2007). Also, the SEDs of these two silicate carbon stars show much larger infrared excesses longward of 3-4 $\mu \mathrm{m}$ (see Fig. 5 in Ohnaka et al. 2006; and Deroo et al. 2007) than BM Gem. This is consistent with the presence of optically thick disks, which is confirmed by the radiative transfer modeling of Ohnaka et al. (2006). These differences in mineralogy and optical thickness appear to affect the wavelength dependence of the observed $N$-band visibilities and differential phases, as discussed below.

The $N$-band visibilities observed toward BM Gem shown in Fig. 2a reflect the optically thin silicate emission: the visibilities are the lowest at $\sim 10 \mu \mathrm{m}$, where the flux contribution of the extended silicate emission is the highest. The baseline dependence of the visibilities observed at $\$ 9 \mu \mathrm{m}$ and at $\gtrsim 11 \mu \mathrm{m}$ is not significant in the baseline range from 44 to $62 \mathrm{~m}$, given the errors in the measurements. This implies that the extended silicate emission is resolved out completely and the sampled visibility component corresponds to the unresolved central star (its angular diameter is estimated to be $\sim 2$ mas as explained below), for which the visibility is constant for the baselines used in our MIDI observations. In this case, the observed visibilities represent the fractional flux contribution from the star. On the other hand, the visibilities observed between 9 and $11 \mu \mathrm{m}$ increase with baseline length from $44-46 \mathrm{~m}$ to $62 \mathrm{~m}$, as can be seen in the data \#1, \#2, and \#4 taken at roughly the same position angle. Therefore, the observed visibilities contain some information about the geometry of the silicate emitting region and are modeled in the next section.

Figure $2 \mathrm{~b}$ shows the calibrated differential phases of BM Gem. Significant non-zero differential phases $\left(\sim-70^{\circ}\right.$ at $10 \mu \mathrm{m})$ are detected in the central part of the silicate emission between $\sim 9$ and $11 \mu \mathrm{m}$ in the data sets obtained on the shortest baselines (\#1 and \#3), while the differential phases observed on the longer baselines (\#2 and \#4) are close to zero. As shown in Fig. 2c, the differential phases derived from the calibrators for the data sets \#1 and \#3 are close to zero. The phases of two calibrators for the data set $\# 3$ are in agreement within $\pm 5^{\circ}$, which we assume to be the error in the observed differential phases (the phase above $12 \mu \mathrm{m}$ is more uncertain due to the increasing effect of the atmospheric water vapor). This confirms that the non-zero differential phases observed toward BM Gem are real and uncover a photocenter shift in the silicate feature caused by an asymmetric circumstellar environment. The non-zero values are observed only in the central part of the silicate feature, although one might expect the differential phase to deviate from zero over the entire feature. However, as explained above, the silicate emission is resolved out completely at $\lesssim 9 \mu \mathrm{m}$ and $\gtrsim 11 \mu \mathrm{m}$ even on the shortest baselines. The visibilities observed at these wavelengths correspond to the unresolved, and therefore, symmetric star. This is why differential phases are almost zero at these wavelengths, as well as in the data taken with the longer baselines.

The wavelength dependence of the $N$-band visibilities observed for BM Gem is in marked contrast to that previously observed toward IRAS 08002-3803 and IRAS 18006-3213 with MIDI by Ohnaka et al. (2006) and Deroo et al. (2007), respectively. The $N$-band visibilities of these two objects show an increase from 8 to $\sim 10 \mu \mathrm{m}$ and remain almost constant or decrease slightly longward of $10 \mu \mathrm{m}$. We note that Deroo et al. (2007) observed non-zero differential phases toward IRAS 18006-3213, which are characterized by a significant phase jump at $\sim 8.3 \mu \mathrm{m}$, completely different from that observed toward BM Gem. Given 

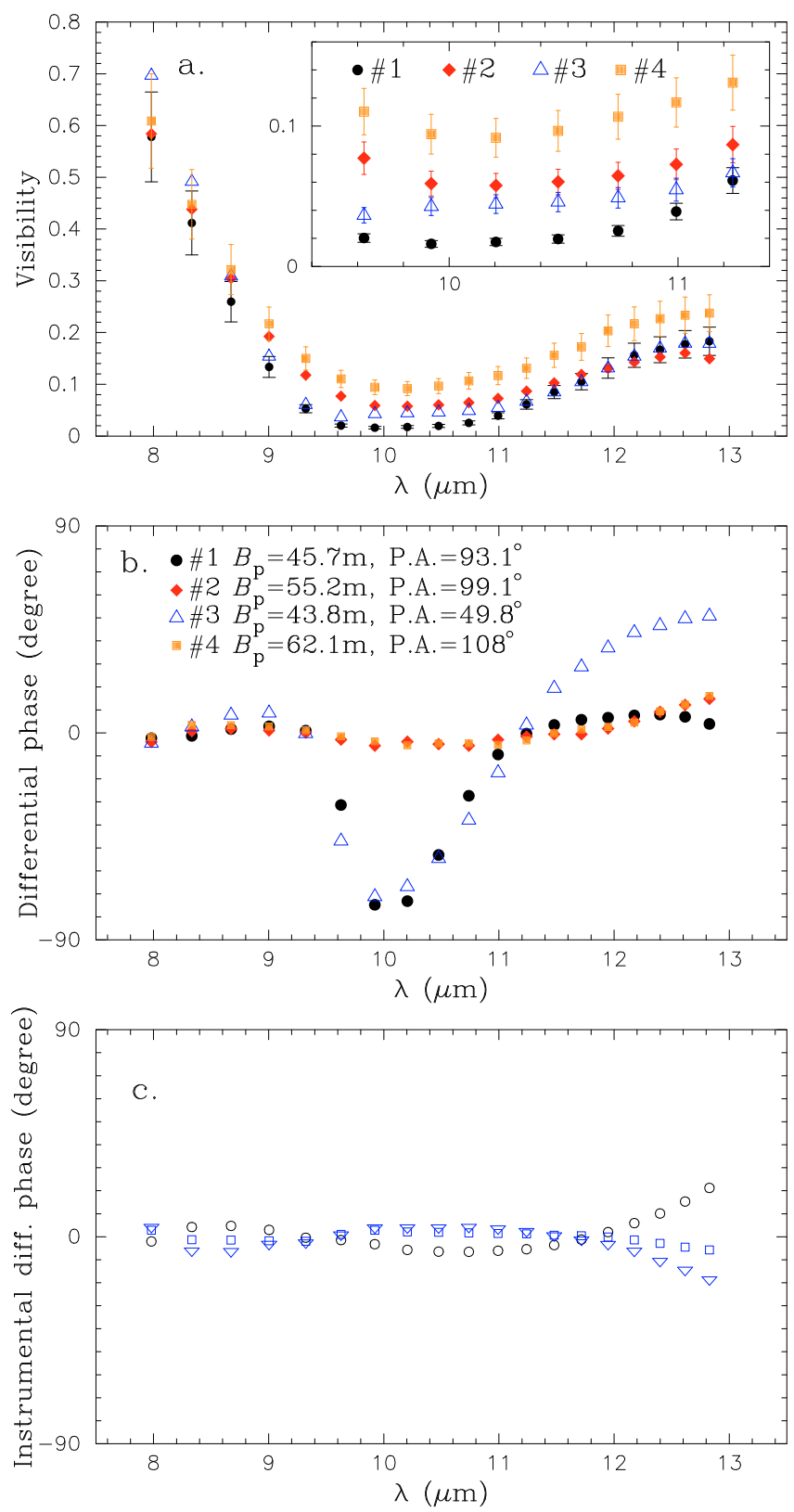

Fig. 2. a) $N$-band visibilities of BM Gem obtained with MIDI. The error bars are shown only for the data sets \#1 and \#4 for visual clarity, but all error bars are drawn in the inset, which shows the enlarged view of the visibilities observed between 9.4 and $11.4 \mu \mathrm{m}$. The data set numbers are defined in Table 1. b) Calibrated differential phases obtained for BM Gem. c) Instrumental differential phases as measured on the calibrators. The open circles represent the phase observed on the same night as the data set \#1, while the open squares and upside-down triangles represent that observed together with \#3.

that the visibility levels observed toward IRAS 18006-3213 range from $\sim 0.1$ to $\sim 0.7$, the difference in the $N$-band visibilities and differential phases cannot be attributed solely to a difference in the object's angular size and/or baselines used in the observations. As outlined above, the dusty environment of BM Gem differs in both mineralogy and optical thickness from that of IRAS 08002-3803 and IRAS 18006-3213. Therefore, the distinct $N$-band visibilities and differential phases observed toward BM Gem most likely reflect the difference in dust chemistry and the structure of the circumstellar environment between BM Gem and IRAS 08002-3803/IRAS 18006-3213.

\section{Geometrical model}

To characterize the asymmetric silicate emitting region, we adopted an "asymmetric-disk" model, in which an unresolved star is surrounded by a ring with a Gaussian radial cross section, and the azimuthal brightness distribution of the ring is sinusoidally modulated. This model was used to interpret the interferometric data of Herbig Ae/Be stars by Monnier et al. (2006). The intensity at the radial distance $r$ from the star and the position angle $\phi$ is expressed as

$$
I_{\lambda}(r, \phi) \propto \mathrm{e}^{-\left(\frac{r-R_{\lambda}}{\sigma_{\lambda}}\right)^{2}} \times\{a \cos (\phi-\Phi)+1\}
$$

where $R_{\lambda}$ and $\sigma_{\lambda}$ denote the radius and the Gaussian width of the ring, respectively. The intensity contrast between the brightest and faintest points of the ring is determined by the parameter $a$, and $\Phi$ is the position angle of the brightest point. $R_{\lambda}$ and $\sigma_{\lambda}$ are dependent on wavelength, but they cannot be well constrained by the current MIDI data at $\lesssim 9 \mu \mathrm{m}$ and $\gtrsim 11 \mu \mathrm{m}$, because the extended component is resolved out at these wavelengths. To take into account that the emission at the longer wavelengths originates in the cooler, outer region, we assumed that the radius is expressed as

$R_{\lambda}=R_{10 \mu \mathrm{m}} \times\left(\frac{\lambda}{10}\right)^{p}$

where $R_{10} \mu \mathrm{m}$ denotes the ring radius at $10 \mu \mathrm{m}$. The ratio between $R_{\lambda}$ and $\sigma_{\lambda}$ was assumed to be independent of wavelength. Therefore, the free parameters of the model are $R_{10 \mu \mathrm{m}}, p$, the ratio $\sigma_{\lambda} / R_{\lambda}, a$, and $\Phi$.

We first derived the ratio of the stellar flux to the total flux at each wavelength as follows. If we approximate the stellar radiation with a blackbody of effective temperature $T_{\text {eff }}(3000 \mathrm{~K}$ for BM Gem, Ohnaka \& Tsuji 1999), the observed flux $F_{\lambda}^{\text {obs }}$ (star + extended dust emission) is given by

$F_{\lambda}^{\mathrm{obs}}=\theta_{\star}^{2} B_{\lambda}\left(T_{\mathrm{eff}}\right) / f_{\lambda}^{\star}$,

where $\theta_{\star}$ and $f_{\lambda}^{\star}$ are the stellar angular radius and the ratio of the stellar flux to the total flux, respectively. As mentioned above, the visibilities at $\lesssim 9 \mu \mathrm{m}$ and $\gtrsim 11 \mu \mathrm{m}$ represent the fractional flux contribution of the star. Using the observed $8 \mu \mathrm{m}$ visibility for $f_{\lambda}^{\star}$ and the observed $8 \mu \mathrm{m}$ flux, $\theta_{\star}$ was derived to be 1.1 mas. Using this $\theta_{\star}$ and the MIDI spectrum, we determined $f_{\lambda}^{\star}$ for all wavelengths in the $N$ band. We note that, as Fig. 1c shows, the SED of BM Gem shortward of $\sim 5 \mu \mathrm{m}$ is well fitted by a blackbody of $3000 \mathrm{~K}$ : this suggests the absence of hot dust, which would have manifested itself as an additional (unresolved) component in our MIDI observations. This verifies our assumption that the observed visibility at $8 \mu \mathrm{m}$ represents the fractional flux of the star.

The intensities of the point-source-like star and the extended component at each wavelength were set to ensure that the flux ratio is consistent with the derived $f_{\lambda}^{\star}$. The visibility amplitude and phase were calculated from the two-dimensional complex visibility computed at each wavelength. The differential phase was calculated in the same manner as the EWS software derives it from MIDI data: a least-square-fit with a straight line (i.e. a linear function with respect to wavenumber) was performed on the model phase as a function of wavenumber. The differential phase was then derived by subtracting the fitted linear component from the model phase. The sign of differential phase measured with MIDI is defined as described in Ratzka et al. (2008). In the model fitting, we excluded the observed differential phase data above $12 \mu \mathrm{m}$ because of the larger systematic uncertainties. 

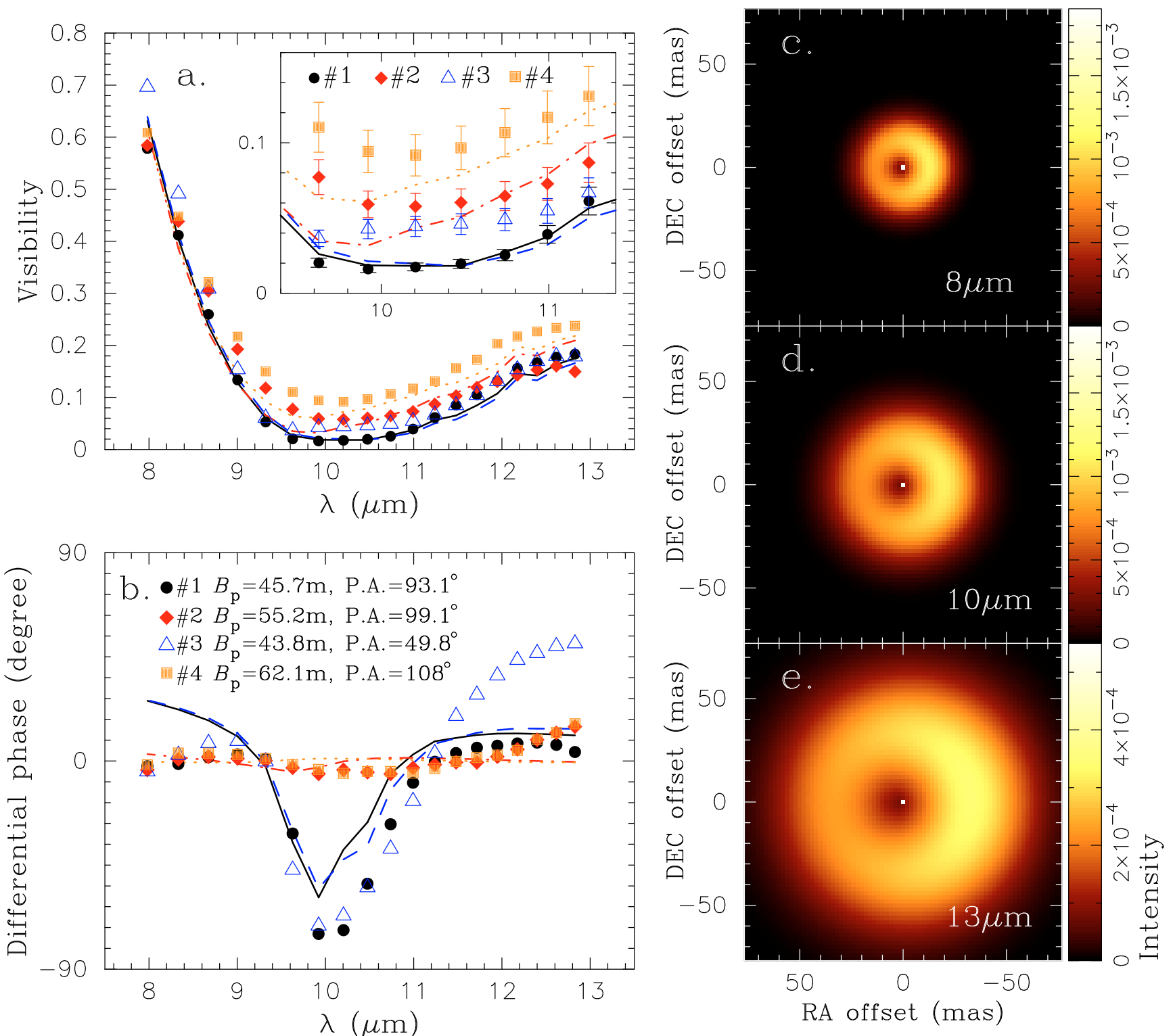

Fig. 3. a) $N$-band visibilities of BM Gem obtained with MIDI (symbols) and those predicted by the best-fit asymmetric-disk model discussed in Sect. 4 (lines). The error bars are shown only in the inset, which shows the enlarged view of the visibilities observed between 9.4 and $11.4 \mu \mathrm{m}$. The data set numbers are defined in Table 1 . The model visibilities are shown by the solid, dashed-dotted, dashed, and dotted lines for the data sets \#1, \#2, \#3, and \#4, respectively, with the same colors as the corresponding data sets. b) Calibrated differential phases obtained for BM Gem (symbols) and those predicted by the asymmetric-disk model (lines) shown in the same manner as in the panel a). The dashed-dotted and dotted lines (red and orange lines) are almost entirely overlapping. c)-e) 8, 10, and $13 \mu \mathrm{m}$ images predicted by the best-fit model. The radius of the ring is 20 mas with a Gaussian radial cross section with a $F W H M$ of 27 mas at $10 \mu \mathrm{m}$. The azimuthal intensity modulation is characterized by $a=0.2$ and $\Phi=280^{\circ}$. The image at each wavelength is normalized so that the integrated flux is 1 (the color on the central star is saturated). A color version of this figure is available in the electronic edition.

Figures $3 \mathrm{a}$ and $\mathrm{b}$ show a comparison between the best-fit model and the MIDI data. This model is characterized by a broad ring with a radius of 20 mas and a Gaussian radial cross section with a FWHM of 27 mas at $10 \mu \mathrm{m}\left(\sigma_{\lambda} / R_{\lambda}=0.8\right)$. The wavelength dependence of the ring radius is characterized by $p=2$, which results in overall sizes of $\sim 45,70$, and 120 mas at 8,10 , and $13 \mu \mathrm{m}$, respectively, as shown in Figs. 3c-e. The intensity of the ring has a mild azimuthal modulation with $a=0.2$, and the brightest point is offset by 20 mas (at $10 \mu \mathrm{m}$ ) at a position angle of $280^{\circ}$ with respect to the star. Figure 3 a reveals that the observed $N$-band visibilities are reproduced reasonably by the model. As shown in Fig. 3b, the predicted differential phases also agree fairly with the observed data. The model predicts the non-zero differential phase values to be too large at $\lesssim 9 \mu \mathrm{m}$ and too small at $\gtrsim 9 \mu \mathrm{m}$ compared with the observed data. Also, the model visibility for the data set \#3 is lower than observed. However, given the simple nature of the model, the fit can be regarded to be good. The $\chi^{2}$ value is 3.8 for this best-fit model. The formal errors in the ring radius at $10 \mu \mathrm{m}\left(R_{10 \mu \mathrm{m}}\right), p$, the ratio $\sigma_{\lambda} / R_{\lambda}$, azimuthal brightness modulation $(a)$, and the position angle of the brightest point $(\Phi)$ are \pm 2 mas, $\pm 0.5, \pm 0.05$, \pm 0.1 , and $\pm 40^{\circ}$, respectively. However, it should be kept in mind that the extended silicate emission is nearly resolved out by our MIDI observations, which makes it difficult to glean information about the overall geometry of the silicate emitting region. Therefore, the above model parameters should be regarded as 
preliminary despite the small formal errors, until they are confirmed by future MIDI observations with a better $(u, v)$ coverage.

\section{Discussion}

Given the spectroscopic signatures of a circum-companion accretion disk discovered toward BM Gem by Izumiura (2003) and Izumiura et al. (2008), it is tempting to interpret the above model as a circum-companion disk. In the scenario of Yamamura et al. (2000), the radiation pressure from the primary star drives an outflow from the circum-companion disk. Depending on the orbital period of the binary system and the outflow velocity, the material blown away from the disk may trail like a cometary tail and form a spiral structure surrounding the entire binary system. The material surrounding the entire system (corresponding to the "cometary tail") can be represented by the broad ring of the asymmetric-disk model, while the circum-companion disk (the "coma" of the cometary trail) appears as the offset bright region. In our model, the offset of the bright feature is dependent on wavelength as the ring's radius, although the position of the companion is independent of wavelength. However, since the mid-infrared emission reflects the distribution of dust, not exactly the companion itself, such wavelength dependence of the offset may also be expected.

If the above model represents the circum-companion disk, the offset of the bright feature from the central star, 13-34 mas, corresponds to the angular separation between the primary star and the companion. This favors a main-sequence star companion rather than a white dwarf companion, because the luminosity from an accretion disk around a white dwarf at separations of 13-34 mas (16-41 AU at a distance of $1.2 \mathrm{kpc})$ is estimated to be $\sim 5-18 L_{\odot}$ in the Bondi-Hoyle formulation (Eq. 5 in Izumiura et al. 2008). This is significantly higher than the observationally derived value of $\sim 0.2 L_{\odot}\left(0.03-0.6 L_{\odot}\right.$, Izumiura et al. 2008). However, it should be noted that the Bondi-Hoyle-type accretion could overestimate the accretion rate as much as by an order of magnitude (e.g. Nagae 2004, and references therein). In this case, the predicted accretion luminosity is $\sim 0.5-1.8 L_{\odot}$, which is marginally compatible with the observed range. Therefore, a main-sequence star companion seems more likely, although a white dwarf companion cannot be entirely excluded.

\section{Conclusion}

Our VLTI/MIDI observations of the silicate carbon star BM Gem have revealed that the $N$-band visibilities are characterized by a steep decrease from 8 to $\sim 10 \mu \mathrm{m}$ and a gradual increase longward of $10 \mu \mathrm{m}$, reflecting the optically thin $10 \mu \mathrm{m}$ emission feature emanating from sub-micron-sized amorphous silicate grains. Furthermore, we have detected significant non-zero differential phases $\left(\sim-70^{\circ}\right)$ between $\sim 9$ and $11 \mu \mathrm{m}$, which reveals the asymmetric nature of the silicate emitting region. The wavelength dependence of the observed $N$-band visibilities and differential phases is remarkably different from that previously observed toward two silicate carbon stars, IRAS 08002-3803 and IRAS 18006-3213. This most likely reflects the differences in mineralogy, optical thickness, and geometry (circumbinary or circum-companion) of the disks.

Our simple geometrical modeling of the observed visibilities and differential phases suggests the presence of a bright region offset from the central star by $13-34$ mas (16-41 AU at $1.2 \mathrm{kpc})$ at a position angle of $280 \pm 40^{\circ}$. This bright region can be interpreted as a circum-companion disk. The derived offset from the central star makes a white dwarf companion unlikely (thus leaving a main-sequence star companion more favorable), because a white dwarf companion at these separations would produce too much accretion luminosity compared to that observed.

However, the lack of detailed information about the geometry of the silicate dust distribution means that this circumcompanion disk interpretation is not yet definitive. For example, we cannot entirely exclude the possibility that the asymmetricdisk model represents a circumbinary disk with an asymmetric structure. MIDI observations with shorter baselines are indispensable for placing tighter constraints on the size and shape of the silicate emitting region. Also, high-resolution imaging at violet/blue wavelengths is important for direct detection of the circum-companion accretion disk. Combination of the violet/blue high-resolution imaging and MIDI observations will provide definitive confirmation that silicate dust is stored in a circum-companion disk.

Acknowledgements. We thank the ESO VLTI team on Paranal and in Garching and the MIDI team for carrying out the observations and making the data reduction software publicly available. We also thank the referee, $\mathrm{H}$. van Winckel, for his constructive comments.

\section{References}

Chan, S. J. 1993, AJ, 106, 2126

Chesneau, O., Meilland, A., Rivinius, T., et al. 2005, A\&A, 435, 275

Cohen, M., Walker, R. G., \& Witteborn, F. C. 1992, AJ, 104, 2030

Cutri, R. M., Skrutskie, M. F., Van Dyk, S., et al. 2003, The 2MASS All-Sky Catalog of Point Sources

Deroo, P., Van Winckel, H., Verhoelst, T., et al. 2007, A\&A, 467, 1093

Egan, M. P., Price, S. D., Kraemer, K. E., et al. 2003, The Midcourse Space Experiment Point Source Catalog Version 2.3, Air Force Research Laboratory Technical Report AFRL-VS-TR-2003-1589

Engels, D. 1994, A\&A, 285, 497

Engels, D., \& Leinert, Ch. 1994, A\&A, 282, 858

ESA 1997, The Hipparcos and Tycho Catalogues, ESA SP-1200

Izumiura, H. 2003, Ap\&SS, 283, 189

Izumiura, H., Noguchi, K., Aoki, W., et al. 2008, ApJ, 682, 499

Jaffe, W. 2004, SPIE Proc., 5491, 715

Leinert, Ch., van Boekel, R., Waters, L. B. F. M., et al. 2004, A\&A, 423, 537

Little-Marenin, I. R. 1986, ApJ, 307, L15

Lloyd-Evans, T. 1990, MNRAS, 243, 336

Monnier, J. D., Berger, J.-P., Millan-Gabet, R., et al. 2006, ApJ, 647, 444

Morris, M. 1987, PASP, 99, 1115

Nagae, T., Oka, K., Matsuda, T., et al. 2004, A\&A, 419, 335

Nakada, Y., Izumiura, H., Onaka, T., et al. 1987, ApJ, 323, L77

Noguchi, K., Kawara, K., Kobayashi, Y., et al. 1981, PASJ, 33, 373

Ohnaka, K., \& Boboltz, D. A. 2008, A\&A, 478, 809

Ohnaka, K., \& Tsuji, T. 1999, A\&A, 345, 233

Ohnaka, K., Driebe, T., Hofmann, K.-H., et al. 2006, A\&A, 445, 1015

Ohnaka, K., Driebe, T., Weigelt, G., \& Wittkowski, M. 2007, A\&A, 466, 1099

Ohnaka, K., Driebe, T., Hofmann, K.-H., Weigelt, G., \& Wittkowski, M. 2008, A\&A, 484, 371

Przygodda, F., Chesneau, O., Graser, U., Leinert, Ch., \& Morel, S. 2003, Ap\&SS, 286, 85

Ratzka, T., et al. 2008, in prep.

Smith, B. J., Price, S. D., \& Baker, R. I. 2004, ApJS, 154, 673

Szczerba, R., Szymczak, M., Babkovskaia, N., et al. 2006, A\&A, 452, 561

Szymczak, M., Szczerba, R., \& Chen, P. S. 2001, in Post-AGB Objects as a Phase of Stellar Evolution, ed. R. Szczerba, \& S. K. Górny, Ap\&SS Library, 265, 439

Willems, F., \& de Jong, T. 1986, ApJ, 309, L39

Yamamura, I., Dominik, C., de Jong, T., Waters, L. B. F. M., \& Molster, F. J. 2000, A\&A, 363, 629 